\title{
High-Resolution Optical and Infrared Observations of Molecules in Comets
}

\author{
S. J. Kim
}

Department of Astronomy and Space Science, Kyunghee University, Suwon 449-701, Korea

Y. C. Minh and S. Hyung

Korea Astronomy Observatory, Yusong, Taejon 305-348, Korea

Y. H. Kim

Department of Astronomy and Space Science, Chungnam National University, Yusong, Taejon 305-764, Korea

\begin{abstract}
Molecules in cometary nuclei are considered to contain information on the constituents in the proto-solar nebula at the time of solar system formation. The composition of cometary nuclei has been indirectly derived from spectroscopic observations of molecules in comae. Ambitious space missions to cometary nuclei for landing and sample return are planned to be realized in 4-7 years. Until then, spectroscopic observations remain a powerful way to remotely investigate molecules and ions in cometary comae. Recent bright comets such as Hale-Bopp (C/1995 O1) and Hyakutake (C/1996 B2) have provided detections of new molecules in comae. Observational results from high-resolution optical and infrared spectroscopy of Hale-Bopp, Hyakutake and recent comets, are presented. In particular, symmetric molecules, which have zero electric dipole moment and are therefore not observable in the radio range, have been detected in several recent comets by infrared observations. The new data can also be used to derive accurate abundances of known molecular species. We present the time-dependent excitation processes of the bands of these molecules and discuss the influence of the fluorescence calculations on the derivations of molecular abundances in comets.
\end{abstract}

\section{Introduction}

Spectroscopic observations of comets from ground-based observatories since Swings et al. (1941) have greatly extended the human knowledge of the constituents of comets. Molecules observed in the comae have been utilized to infer their abundances in the nuclei. Parent molecules in the cometary nuclei are thought to have information about constituents in the pre-solar nebula at the time of solar system formation, or information on the molecular abundances of local interstellar clouds. During the last five decades, the spectral resolving power of spectrometers in the visible range for cometary spectroscopy has gradually 
increased from $\lambda / \Delta \lambda \sim 1,000$ (Swings et al. 1941; Dossin et al. 1961) to $\sim 100,000$ (Brown et al. 1996) nowadays. Spectroscopic observations in the visible range (3000-9000 $\AA$ ) have thus far detected radicals, such as $\mathrm{OH}, \mathrm{NH}, \mathrm{CH}, \mathrm{CN}, \mathrm{C}_{2}$, $\mathrm{C}_{3}, \mathrm{NH}_{2}$ (e.g. Arpigny et al. 1991; Brown et al. 1996); ions, such as $\mathrm{CO}^{+}, \mathrm{OH}^{+}$, $\mathrm{CH}^{+}, \mathrm{H}_{2} \mathrm{O}^{+}, \mathrm{N}_{2}^{+}, \mathrm{CO}_{2}^{+}$(e.g. Wyckoff et al. 1996); and only one stable molecule, $\mathrm{S}_{2}$ (Kim et al. 1990). Stable molecules, which may be parent to radicals and ions detected in comae, are usually detected in infrared and radio ranges. Highresolution spectroscopy in the visible range makes it possible to investigate the detailed fluorescence and collisional excitation processes of the radicals and ions in the comae (e.g. Schleicher et al. 1982; Kim et al. 1989), and to separate isotopic molecular lines from adjacent normal molecular lines (e.g. Kleine et al. 1994, 1995).

Infrared observations of comets started with low-resolution spectroscopy mainly looking for continuum emission of dust and ice (e.g. Tokunaga et al. 1984), but soon $\mathrm{P} /$ Halley provided a justification to use a high resolution $(\lambda / \Delta \lambda$ $=90,000)$ Fourier Transform Spectrometer on the NASA/Kuiper Airborne Observatory (KAO) to detect $2.65 \mu \mathrm{m} \mathrm{H}_{2} \mathrm{O}$ lines (e.g. Mumma et al. 1986). The IKS/Vega experiment detected $\mathrm{H}_{2} \mathrm{CO}, \mathrm{CO}_{2}$, and possibly $\mathrm{CO}$ in addition to $\mathrm{H}_{2} \mathrm{O}$ in the 2.5-5 $\mu \mathrm{m}$ region of $\mathrm{P} / \mathrm{Halley}$ (Moroz et al. 1987). Subsequently groundbased infrared observations positively detected $\mathrm{CH}_{3} \mathrm{OH}$ in Comet P/Swift-Tuttle (Hoban et al. 1993).

Recent bright comets, Hale-Bopp (C/1995 O1) and Hyakutake (C/1996 B2), provided other opportunities to detect the infrared lines of parent molecules unambiguously, such as $\mathrm{CH}_{4}, \mathrm{C}_{2} \mathrm{H}_{6}, \mathrm{CO}$ (e.g. Mumma et al. 1996), $\mathrm{C}_{2} \mathrm{H}_{2}$ (e.g. Brooke et al. 1996), HCN (e.g. Weaver et al. 1999a), and OCS (Dello Russo et al. 1998). Hale-Bopp and Hyakutake also yielded high signal-to-noise spectra of radicals in the visible region (Cochran et al. 1997; Meier et al. 1998). In this paper, we review the development of the understanding of cometary molecules as the spectral resolution has increased during the last two decades. The detections of cometary molecules are chronologically presented. The recent detections of new molecules in bright comets Hale-Bopp and Hyakutake in the infrared region are highlighted. Implications of the molecular abundances and isotopic ratios in comets are discussed. The need for time-dependent fluorescence calculations of cometary molecules is emphasized, and some of the recent results are presented.

\section{Optical Range}

In the optical range, few parent molecules are seen with the exception of $\mathrm{S}_{2}$ (Kim et al. 1990). Most of the emission lines are due to atoms or dissociative fragments from parent molecules, ions, and isotopic molecules. Therefore, the optical region is not a good wavelength range to study the parent molecules, but the wealth of emission lines from radicals makes it a good place to investigate detailed fluorescence processes and to study their isotopic ratios. Brown et al. (1996) presented high-resolution spectra $(\lambda / \Delta \lambda \sim 42,000)$ of the comae of comets Swift-Tuttle and Brorsen-Metcalf in the spectral range between 3800 and $9900 \AA$. They identified 2438 lines, which are due to $\mathrm{H}, \mathrm{O}, \mathrm{C}_{2}, \mathrm{CN}, \mathrm{NH}_{2}, \mathrm{C}_{3}$, $\mathrm{H}_{2} \mathrm{O}^{+}, \mathrm{CH}$, and $\mathrm{CH}^{+}$among 2997 emission lines, leaving hundreds of unidentified lines. Ionic emission lines are prominent at ion tails, and Wyckoff et al. 
(1996) also reported unidentified ionic lines in the spectra of the plasma tail of $\mathrm{P} /$ Halley. High-resolution molecular emission in the optical range stimulated modelers to construct synthetic spectra of cometary molecular bands. Schleicher et al. (1982) constructed a fluorescence model of $\mathrm{OH}$ bands which appeared in the IUE high-dispersion spectra of comets in the near ultraviolet range, which can also be observed from ground. The $\mathrm{OH}$ model includes ro-vibrational transitions $\left(v^{\prime}=0-2 \rightarrow v^{\prime \prime}=0-2\right)$ and Swings effects. Kim et al. (1989) constructed a model of the $0-0$ band of the A-X transition of $\mathrm{NH}$, which occurs in the 3345$3375 \AA$ range of $\mathrm{P} /$ Halley spectra. They found that the $\mathrm{NH}$ spectrum is due to pure fluorescence with negligible influence by collisions, suggesting that most of the $\mathrm{NH}$ molecules exist further from the $\mathrm{P} / \mathrm{Halley}$ nucleus than $30,000 \mathrm{~km}$, and that there should be sources in addition to $\mathrm{NH}_{3}$ from the nucleus. Gredel et al. (1989) updated a previous $\mathrm{C}_{2}$ model of $\mathrm{A}^{\prime}$ Hearn (1978) including detailed rotational transitions of electronic bands, which consist of more than 4,000 rotational quantum states. Their model was compared with the high resolution spectra of $\mathrm{P} /$ Halley, and the fit looks satisfactory. However, the authors pointed out that accurate determinations of the transition probabilities of the electronic bands should improve their model significantly. Kleine et al. (1994) presented computed flourescence spectra of the stable isotopes of $\mathrm{CN}$, such as ${ }^{12} \mathrm{C}^{14} \mathrm{~N},{ }^{13} \mathrm{C}^{14} \mathrm{~N}$, ${ }^{12} \mathrm{C}^{15} \mathrm{~N}$, in the comae of comets. In the model spectra, they considered Swings effects including collisional effects, which are shown to be significant in the fluorescence process. Their computed spectra are compared with high-resolution spectra of comets $\mathrm{P} /$ Halley, Austin, and Levy.

Kleine et al. (1995) derived a ${ }^{13} \mathrm{CN} /{ }^{12} \mathrm{CN}$ isotope ratio of $95 \pm 12$ analyzing high-resolution spectra of $\mathrm{P} / \mathrm{Halley}$. The result agrees with the solar carbon isotope abundance ratio of 90 . This agreement suggests that comets should be co-evolved from the same molecular cloud as other members of the solar system. Kim et al. (1997) analyzed the high resolution spectra of the A-X band $(\sim 4300$ $\AA$ ) and B-X band $(\sim 3890 \AA)$ of CH of comet Austin $(1990 \mathrm{~V})$. They found that the $\mathrm{CH}$ bands do not achieve fluorescence equilibrium because its lifetime is too short ( $\sim 100$ seconds) to equilibrate the quantum state populations of the bands. They also discuss the possible parent molecules of $\mathrm{CH}$ and the long lifetimes of the parent molecules in view of the fact that the $\mathrm{CH}$ emission is extended far from the nucleus despite its short lifetime. Arpigny et al. (1987) previously showed extended emission of $\mathrm{CH}$ up to $10^{5} \mathrm{~km}$ from the nuclei of various comets. Recently, Meier et al. (1998) analyzed high resolution spectra of the $\mathrm{NH}$ and $\mathrm{CH}$ bands of Hyakutake, and derived upper limits on the ND/NH and $\mathrm{CD} / \mathrm{CH}$ ratios, which are $6 \times 10^{-3}$ and $1 \times 10^{-3}$, respectively. Millar et al. (1989) investigated gas-phase chemistry including ion-molecule reactions in dense interstellar clouds in order to derive deuterium fractionations of various molecules. According to their model calculations, the $\mathrm{ND} / \mathrm{NH}$ and $\mathrm{CD} / \mathrm{CH}$ ratios should be in the ranges of $1 \times 10^{-2}-2 \times 10^{-4}$ and $1 \times 10^{-2}-4 \times 10^{-3}$, respectively, depending upon model conditions, and these ratios are significantly greater than the $\mathrm{HD} / \mathrm{H}_{2}$ ratio, which is $\sim 3 \times 10^{-5}$. The upper limit results of Meier et al. (1998) are highly meaningful for further constraining the models of Millar et al., because they are within or even less than the model ratios.

Among the detected neutral radicals in the 3000-9000 $\AA$ range, the lineby-line spectral structures of $\mathrm{NH}_{2}$ and $\mathrm{C}_{3}$ bands have not been modelled in 
detail. $\mathrm{NH}_{2}$ is an asymmetric top molecule, and therefore, its band structure is very complicated compared with other linear and symmetric molecules. While the line-by-line quantum-number designations of the $\mathrm{NH}_{2}$ bands have been carried out by Dressler \& Ramsay (1959), their line-by-line transition probabilities have not been accurately determined thus far. The lack of the accurate transition probabilities prevents the development of a fluorescence model for the $\mathrm{NH}_{2}$ bands. The situation for the $\mathrm{C}_{3}$ bands may be worse. Although Gausset et al. (1965) listed quantum-number designations for strong lines, the list lacks weak line designations, and it does not list transition probabilities for the lines. Since the $\mathrm{NH}_{2}$ and $\mathrm{C}_{3}$ bands are prominent emission structures in the visible range, the modeling of these bands are urgently needed to properly analyze the high-resolution spectra of recent bright comets.

Among the detected ions, only two molecules, $\mathrm{CO}^{+}$and $\mathrm{CO}_{2}^{+}$, have been modeled. Magnani \& A'Hearn (1986) constructed a fluorescence equilibrium model of the visible bands of $\mathrm{CO}^{+}$, including Swings and Greenstein effects as a function of heliocentric radial velocity. Kim (1999) constructed a fluorescence equilibrium model of the $\mathrm{A}-\mathrm{X}$ and $\mathrm{B}-\mathrm{X}$ band systems of $\mathrm{CO}_{2}^{+}$, and compared the model with the spectra of comets Austin (1989c1) and P/Giacobinni-Zinner. The model, however, includes vibrational-vibrational transitions only. Other ionic molecules, such as $\mathrm{OH}^{+}, \mathrm{CH}^{+}, \mathrm{H}_{2} \mathrm{O}^{+}$, and $\mathrm{N}_{2}^{+}$, are awaiting to be modeled to be used for the analyses of plasma tail spectra.

\section{Infrared Range}

Before $\mathrm{P} /$ Halley, low resolution spectroscopy in the infrared region yielded only the continuum emission from dust and/or ice through the 3,10 , and $20 \mu \mathrm{m}$ atmospheric windows (e.g. Tokunaga et al. 1984). Recently, low resolution observations of Hale-Bopp made by the Infrared Space Observatory (ISO) provided a spectacular panorama of the infrared spectrum of dust particles in the $7-45 \mu \mathrm{m}$ range (Crovisier et al. 1997). The spectral structure of the dust spectrum has been found to be remarkably similar to the spectral structure of $\mathrm{Mg}$-rich olivine like forsterite (Crovisier 1998). Therefore, (part of) the dust may be ascribed to crystalline silicates, which are similar to that observed in circumstellar disks of Vega-type stars, but contrast with interstellar silicates that are amorphous.

A high resolution $(\lambda / \Delta \lambda=90,000)$ Fourier Transform Spectrometer on the NASA/Kuiper Airborne Observatory (KAO) successfully resolved the individual lines of the $2.65 \mu \mathrm{m}$ band of $\mathrm{H}_{2} \mathrm{O}$ (Mumma et al. 1986). The high resolution spectra were used to derive the ortho-para ratio of $\mathrm{H}_{2} \mathrm{O}$, which is a valuable quantity to explore the thermal history of the cometary nucleus (Mumma et al. 1987). The low resolution spectrometer, IKS, on Vega also detected molecular emissions from the bands of $\mathrm{H}_{2} \mathrm{CO}, \mathrm{CO}_{2}$, and possibly $\mathrm{CO}$, in addition to the $\mathrm{H}_{2} \mathrm{O}$ band in the 2.5-5 $\mu \mathrm{m}$ region of $\mathrm{P} / \mathrm{Halley}$ (Moroz et al. 1987). After $\mathrm{P} /$ Halley, Hoban et al. (1993) detected emission lines of the $3.52 \mu \mathrm{m}$ band of $\mathrm{CH}_{3} \mathrm{OH}$ at $\mathrm{P} /$ Swift-Tuttle. The abundance of $\mathrm{CH}_{3} \mathrm{OH}$ is found to be $\sim 7 \%$ constituting a significant portion of cometary volatile carbon, and the $3.52 \mu \mathrm{m}$ band makes up $60 \%$ of the $3.4 \mu \mathrm{m}$ 'cometary organic feature.'

The recent bright comets Hale-Bopp and Hyakutake provided opportunities to detect several parent molecules unambiguously with high-resolution infrared 
spectroscopy. In particular, symmetric molecules, such as $\mathrm{CH}_{4}, \mathrm{C}_{2} \mathrm{H}_{6}$, and $\mathrm{C}_{2} \mathrm{H}_{2}$ which have no permanent electric dipole moment, can only be observed in the infrared, and not by radio observations, which have been successful in the detections of many parent molecules that have dipole moments (e.g. Bockelée-Morvan et al. 1991; Crovisier et al. 1991; Biver et al. 1997). These symmetric molecules are not observable in emission in the ultraviolet region either, because they are predissociated by solar ultraviolet radiation before emitting fluorescence light. Mumma et al. (1996) positively detected the infrared lines of $\mathrm{C}_{2} \mathrm{H}_{6}, \mathrm{CH}_{4}$, and $\mathrm{CO}$ in the coma of Hyakutake with the high-resolution CSHELL grating spectrometer at the NASA IRTF (Infrared Telescope Facility). They found an abundant $\mathrm{C}_{2} \mathrm{H}_{6} / \mathrm{CH}_{4}$ ratio, which is consistent with the production of $\mathrm{C}_{2} \mathrm{H}_{6}$ in icy grain mantles in interstallar clouds (Hasegawa \& Herbst 1993), and not consistent with a thermochemically equilibrated region in the proto-solar nebula (e.g. Prinn \& Fegley 1989). Notesco et al. (1997) experimentally demonstrated that the $\mathrm{C}_{2} \mathrm{H}_{6}, \mathrm{CH}_{4}$, and $\mathrm{CO}$ abundances could be obtained by the trapping of these gases in $\mathrm{H}_{2} \mathrm{O}$ ice at $65 \mathrm{~K}$, and discussed the formation of $\mathrm{H}_{2} \mathrm{O}$ ice particles in the outskirts of the interstellar cloud or the proto-solar nebula. Almost at the same time as Mumma et al. (1996), Brooke et al. (1996) observed Hyakutake and detected the $3 \mu \mathrm{m}$ band lines of $\mathrm{C}_{2} \mathrm{H}_{2}$. The same molecular species, $\mathrm{H}_{2} \mathrm{O}, \mathrm{CH}_{4}, \mathrm{C}_{2} \mathrm{H}_{6}, \mathrm{C}_{2} \mathrm{H}_{2}$, and $\mathrm{CO}$, in addition to $\mathrm{HCN}$ were detected in HaleBopp (Weaver et al. 1999a). OCS was first detected in Hyakutake in the radio (Woodney et al. 1997), and subsequently Dello Russo et al. (1998) detected the $4.85 \mu \mathrm{m}$ band lines of OCS in Hale-Bopp. The derived $\mathrm{Q}(\mathrm{OCS}) / \mathrm{Q}\left(\mathrm{H}_{2} \mathrm{O}\right)$ ratios are $3 \times 10^{-3}$ for Hyakutake (Woodney et al. 1997), and $3-5 \times 10^{-3}$ for Hale-Bopp (Dello Russo et al. 1998), which are the most abundant production rates among sulfur-containing molecules, such as $\mathrm{H}_{2} \mathrm{~S}, \mathrm{CS}_{2}, \mathrm{CS}, \mathrm{S}_{2}, \mathrm{SO}_{2}, \mathrm{SO}$, and $\mathrm{H}_{2} \mathrm{CS}$.

Recently, Weaver et al. (1999b) observed comet $21 \mathrm{P} / \mathrm{GZ}$ and found that $\mathrm{C}_{2} \mathrm{H}_{6}$ is depleted at least by a factor of $\sim 10$ compared to its relative abundance in Hale-Bopp and Hyakutake. This depletion is similar to that observed for $\mathrm{C}_{2}$ and $\mathrm{C}_{3}$ from optical observations of GZ (A'Hearn et al. 1995), suggesting that the formation of volatile carbon-chain molecules was inhibited in GZ. They, however, could not find any clear correlation between the $\mathrm{C}_{2} \mathrm{H}_{6}$ and $\mathrm{C}_{2} / \mathrm{C}_{3}$ abundances in a sample of nine other comets. Around the same time, Mumma et al. (1999) also observed comet $21 \mathrm{P} / \mathrm{GZ}$, and found that $\mathrm{C}_{2} \mathrm{H}_{6}$ is substantially less abundant compared with Hale-Bopp and Hyakutake, suggesting a heliocentric gradient in the $\mathrm{C}_{2} \mathrm{H}_{6}$ mixing ratio in precometary ices in the proto-solar nebula. Weaver et al. (1999c) observed C/Lee $(1999 \mathrm{H} 1)$ and detected the emission lines of the $\nu_{7}$ band of $\mathrm{C}_{2} \mathrm{H}_{6}$ near $3.35 \mu \mathrm{m}$ and possibly the $\nu_{5}$ band of $\mathrm{C}_{2} \mathrm{H}_{6}$ near $3.45 \mu \mathrm{m}$.

\section{Excitation and De-Excitation Processes of Cometary Molecules}

In order to analyze the high-resolution molecular spectra of comets, we need to calculate the quantum state populations of the molecules. When a comet approaches the sun, as the temperature of the nucleus increases, the comet releases volatiles continuously forming a coma. The released molecules in the coma are immediately exposed to solar radiation undergoing excitation and deexcitation processes in the quantum states. These fluorescence processes yield unique line emission of particular species, which have their own special structures 

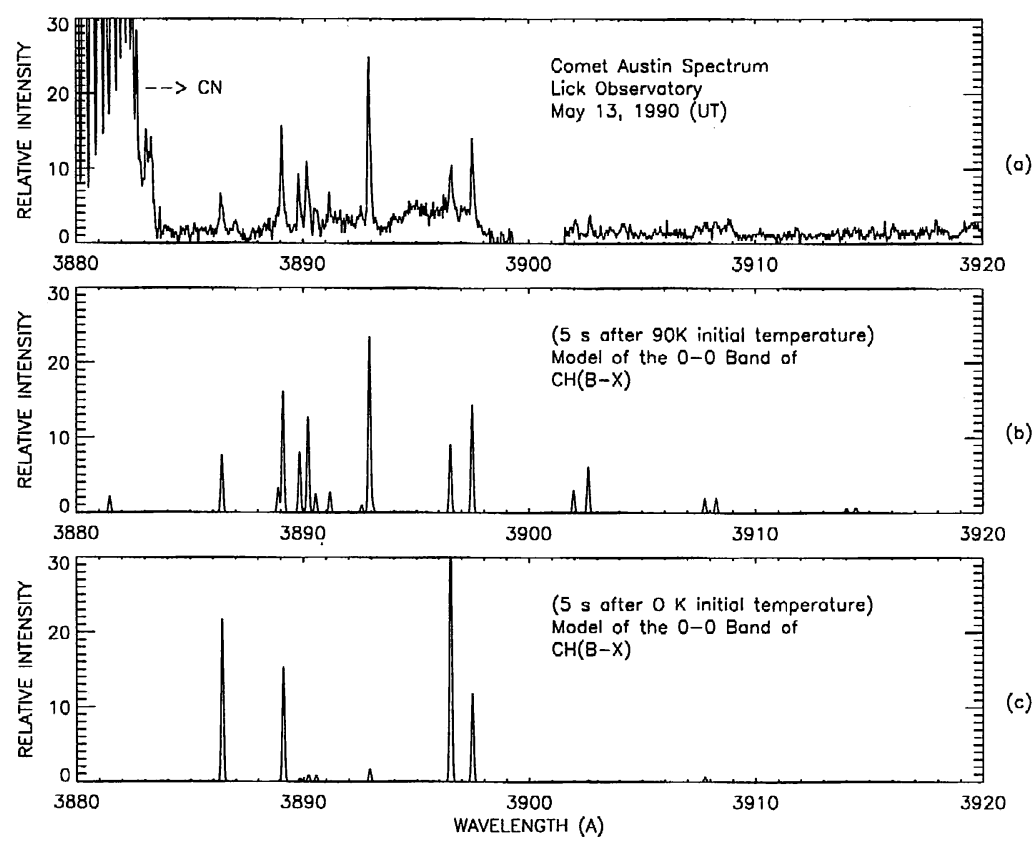

Figure 1. (a) High resolution spectrum of comet Austin. Because of a gap between the orders of the echelle spectrometer, spectral data between 3899-3902 $\AA$ are absent. (b) and (c) show the B-X band models of $\mathrm{CH}$ after 5 seconds exposure to sunlight with initial temperatures of $90 \mathrm{~K}$ and $0 \mathrm{~K}$, respectively. Comparison with the observations of comet Austin shows that the nascent temperature of $\mathrm{CH}$ should be $\sim 90 \mathrm{~K}$. The Austin spectrum was obtained by Kim, Brown, \& Spinrad (1997).

from extreme ultraviolet to radio range. Thus far, no absorption structures in the cometary continuum have been detected, although certain comets, such as $\mathrm{P} /$ Halley and Hale-Bopp are very dusty having bright spectral continuum. The fractional population, $x_{i}$, of the quantum states can be calculated by solving the population evolution equations. The temporal rate of the fractional population is given by:

$$
\frac{d x_{i}}{d t}=-x_{i} \sum_{j}\left(A_{i j}+B_{i j} \rho_{i j}+C_{i j}\right)+\sum_{j} x_{j}\left(A_{j i}+B_{j i} \rho_{j i}+C_{j i}\right)
$$

where $A$ is the Einstein $A$ coefficient for spontaneous emission; $B$ is the Einstein $B$ coefficient for absorption; $\rho$ is the solar flux density; $B \rho$ is the absorption transition rate; and $C$ is the transition rate by collisions with neutrals and ions. The first term in Eq. (1) represents the sum of outgoing population rates from the quantum state $i$ to $j$, and the second term represents the sum of incoming population rates from the state $j$ to $i$, wherever transitions are possible. Eq. (1) can be expressed by a simple first order linear differential equation, 

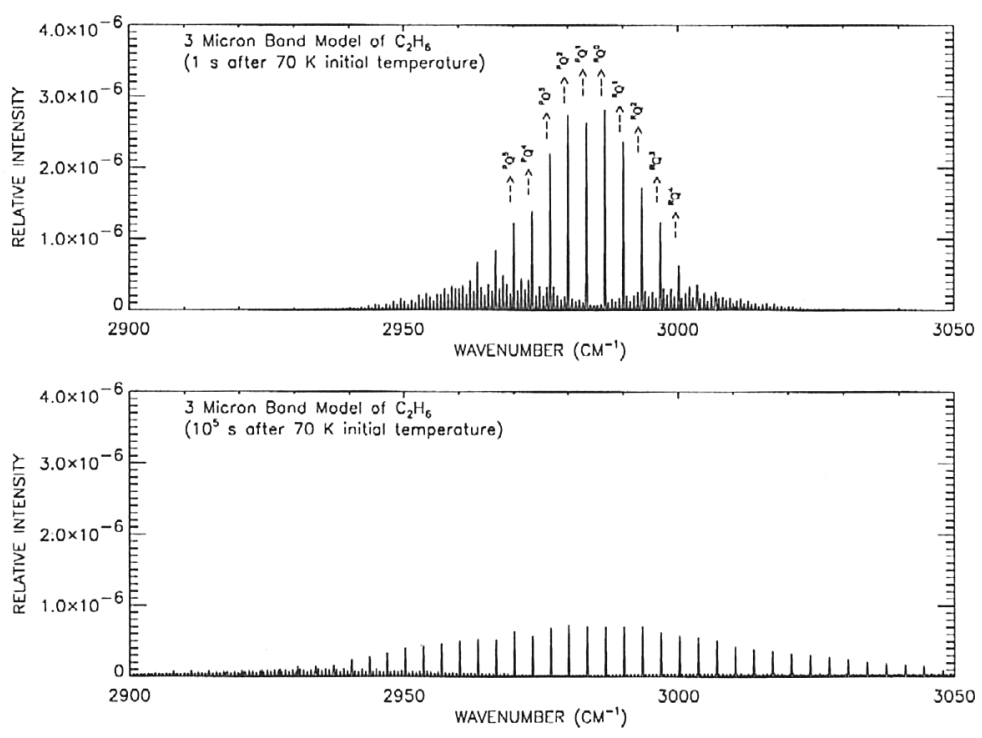

Figure 2. (a) Model spectrum of the $\nu_{7}$ band of $\mathrm{C}_{2} \mathrm{H}_{6}$ immediately after the release from the nucleus with a nascent temperature of 70 $\mathrm{K}$. (b) Model spectrum of the same band $10^{5}$ seconds after the release from the nucleus. The model spectrum in (b) does not depend on the initial temperature of $\mathrm{C}_{2} \mathrm{H}_{6}$, suggesting that after $10^{5}$ seconds exposure to sunlight, the $\nu_{7}$ band achieves fluorescence equilibrium.

$$
\frac{d x}{d t}=\mathrm{A} \cdot \mathrm{x}
$$

where $A$ is the matrix of the coefficients, and $x$ is the vector of fractional populations. The coefficients in A usually differ by many orders of magnitude, and the equations, in principle, require a short time step $\left(\mathrm{ex} . \sim \mathrm{A}_{i j}^{-1}\right)$ to be solved numerically. Traditionally these kind of stiff differential equations have been approximately solved by employing a numerical method developed by Gear (1971), and other improved methods listed in Press et al. (1992). Kim et al. (1990) developed a different method to approximately solve the stiff differential equations for the fluorescence processes of the ultraviolet bands of $S_{2}$. Laffont \& Boice (1997) used the Gear method and compared their results with those of Kim et al. (1990). The results of the detailed comparisons can be found in Laffont \& Boice (1997) and Kim et al. (1999). Recently, Hairer \& Wanner (1996) revisited the stiff case, and constructed their own computer codes for solving the stiff equations. We compared the $\mathrm{S}_{2}$ result from the computer codes of Hairer \& Wanner (priv. comm. with E. Hairer 1999) with those from Laffont \& Boice (1997) and found that the results are the same. If the quantum state populations are in equilibrium, Eq. (2) becomes,

$$
A \cdot x=0
$$


Short-lived molecules do not achieve fluorescence equilibrium during their lifetimes. Some long-lived molecules do not achieve fluorescence equilibrium either, if they have strict selection rules. Although one can use the fluorescence equilibrium approximation in many cases, time-dependent fluorescence calculations are often necessary to analyze cometary spectra for short-lived molecules or for molecules having strict selection rules.

For example, the lifetimes of $\mathrm{CH}, \mathrm{S}_{2}$, and $\mathrm{C}_{2} \mathrm{H}_{6}$ against photodissociation are approximately 100,500 , and $10^{5}$ seconds, respectively, at $1 \mathrm{AU}$ from the Sun. When $\mathrm{S}_{2}$ was first detected in comet IRAS-Araki-Alcock, A'Hearn et al. (1983) thought that the B-X bands of $S_{2}$ seen in IUE spectra may not be in fluorescence equilibrium, since the $S_{2}$ lifetime is short. Using a time-dependent model calculation, Kim et al. (1990), however, demonstrated that the B-X bands are approximately in fluorescence equilibrium after 500 seconds exposure to sunlight, because there is no restriction on the transitions between vibrational states in the $\mathrm{B}-\mathrm{X}$ system. The lifetime of $\mathrm{CH}$ is also very short, but since there are strict selection rules between rotational transitions in the $\mathrm{A}-\mathrm{X}$ and $\mathrm{B}-\mathrm{X}$ systems, it takes more than 1000 seconds to achieve fluorescence equilibrium. Therefore, $\mathrm{CH}$ will be dissociated long before it achieves fluorescence equilibrium. What we see at a comet is the $\mathrm{CH}$ band emissions only 5-10 seconds after they are first exposed to sunlight. Therefore, the $\mathrm{CH}$ band structures may have information on the initial temperature of $\mathrm{CH}$. Fig. 1a shows a high resolution spectrum of comet Austin. Figs. $1 \mathrm{~b}$ and $\mathrm{c}$ show the B-X band models of $\mathrm{CH}$ after 5 seconds exposure to sunlight with initial temperatures of $90 \mathrm{~K}$ and $0 \mathrm{~K}$, respectively. Comparison with the observations of comet Austin clearly show that the nascent temperature of $\mathrm{CH}$ should be approximately $90 \mathrm{~K}$.

The $\nu_{7}$ band of $\mathrm{C}_{2} \mathrm{H}_{6}$ also has a strict selection rule for the rotational transitions. Fig. $2 \mathrm{a}$ shows a model spectrum of the $\nu_{7}$ band of $\mathrm{C}_{2} \mathrm{H}_{6}$ immediately after the release from the nucleus with a nascent temperature of $70 \mathrm{~K}$. Fig. $2 \mathrm{~b}$ shows a model spectrum of the same band $10^{5}$ seconds after the release from the nucleus. The model spectrum in Fig. $2 \mathrm{~b}$ is independent from the initial temperature of $\mathrm{C}_{2} \mathrm{H}_{6}$, suggesting that the $\nu_{7}$ band achieves fluorescence equilibrium after $10^{5}$ seconds exposure to sunlight. As demonstrated in Figs. 1 and 2, the fluorescence efficiencies can be different by factors of 3-4 depending upon the fluorescence conditions, lifetimes, and initial temperatures. Detailed results will be presented by Kim et al. (1999).

\section{Conclusion}

We reviewed the high-resolution observations of comets in the visible and infrared regions during the last two decades, and presented the cosmogonic implications of the obtained abundances of the detected species. We are awaiting recently planned space missions to cometary nuclei for landing and sample return, which will be realized in the next decade. Until then, we will continue to perform high-resolution spectroscopic observations of comets to investigate molecules in cometary comae. We highlighted the recent detections of symmetric parent molecules, which can only be detected in the infrared, and whose abundances are of great importance in constraining currently-available chemical models during the formations of cometary material. We also showed how time- 
dependence affects the calculations of fluorescence efficiencies of molecular lines, and ultimately influence the derivations of the abundances of molecules.

Acknowledgments. We gratefully acknowledge financial support through grant from the Korean Science and Engineering Foundation.

\section{References}

A'Hearn, M.F. 1978, ApJ, 219, 768

A'Hearn, M.F., Feldman, P.D., \& Schleicher, D.G. 1983, ApJ, 274, L99

A'Hearn, M.F., Millis, R.L., Schleicher, D.G., Osip, D.J., \& Birch, P.V. 1995, Icarus, 118, 223

Arpigny, C., Sippen, C.J., Klutz, M., Magain, P., \& Hutsemekers, D. 1987, Symposium on the Diversity and Similarity of Comets, Brussels, Belgium, ESA SP-278, 607

Arpigny, C., et al. 1991, International Conference on Asteroids, Comets, Meteors, Flagstaff, Arizona

Biver, N., et al. 1997, Science, 275, 1915

Bockelée-Morvan, D., Colom, P., Crovisier, J., Despois, D., \& Paubert, G. 1991, Nature, 350, 318

Brooke, T.Y., et al. 1996, Nature, 383, 606

Brown, M.E., Bouchez, A.H., Spinrad, H., \& Johns-Krull, C.M. 1996, ApJ, 112, 1197

Cochran, A.L., Barker, E.S., Cochran, W.D., \& Lambert, D.L. 1997, BAAS, 29, 1050

Crovisier, J. 1998, BAAS, 30, 1059

Crovisier, J., Bockelee-Morvan, D., Colom, P., Despois, D., \& Paubert, G. 1991, Icarus, 93, 246

Crovisier, J., et al. 1997, Science, 275, 1904

Dello Russo, N., Disanti, M.A., Mumma, M.J., Magee-Sauer, K., \& Rettig, T.W. 1998, Icarus, 135, 377

Dressler, K. \& Ramsay, D.A. 1959, Phil. Trans. R. Soc. A, 251, 553

Dossin, F., Fehrenbach, Ch., Haser, L., \& Swings, P. 1961, Ann. Astrophys., 24, 519

Gausset, L., Herzberg, G., Lagerqvist, A., \& Rosen, B. 1965, ApJ, 142, 45

Gear, C.W. 1971, Numerical Initial Value Problems in Ordinary Differential Equations, Prentice Hall, Englewood Cliffs, New Jersey.

Gredel, R., van Dishoeck, E.F., \& Black, J.H. 1989, ApJ, 338, 1047

Hairer, E. \& Wanner, G. 1996, Springer Series in Computational Mathematics 14 , Springer-Verlag

Hasegawa, T.I. \& Herbst, E. 1993, MNRAS, 263, 589

Hoban, S., Reuter, M.A., DiSanti, M., Mumma, M.J., \& Elston, R. 1993, Icarus, 105,548

Kim, S.J. 1999, Earth Planets Space, 51, 139

Kim, S.J., A'Hearn, M.F., \& Cochran, W.D. 1989, Icarus, 77, 98

Kim, S.J., A'Hearn, M.F., \& Larson, S. 1990, Icarus, 87, 440

Kim, S.J., Brown, M., \& Spinrad, H. 1997, J. of Geomagnetism and Geoelectricity, 49, 132

Kim, S.J., Weaver, H.A., Brooke, T.Y., \& Chin, G. 1999, submitted to Icarus

Kleine, M., Wyckoff, S., Wehinger, P.A., \& Peterson, B. 1994, ApJ, 436, 885 
1995, ApJ, 439, 1021

Laffont, C. \& Boice, D.C. 1997, BAAS, 29, 1030

Magnani, L. \& A'Hearn, M.F. 1986, ApJ, 302, 477

Meier, R., Wellnitz, D., Kim, S.J., \& A'Hearn, M. 1998, Icarus, 136, 268

Millar, T.J., Bennett, A., \& Herbst, E. 1989, ApJ, 340, 906

Moroz, W.I., et al. 1987, A\&A, 187, 513

Mumma, M.J., DiSanti, M.A., Dello Russo, N., Magee-Sauer, K., \& Rettig, T.W. 1999, ApJ, in press

Mumma, M.J., Weaver, H.A., \& Larson, H.P. 1987, A\&A, 187, 419

Mumma, M.J., Weaver, H.A., Larson, H.P., Williams, M., \& Davis, D.S. 1986, Science, 232, 1523

Mumma, M.J., et al. 1996, Science, 272, 1310

Notesco, G., Faufer, D., \& Bar-Nun A. 1997, Icarus, 125, 471

Press, W.H., Teukolsky, S.A., Vetterling, W.T., \& Flannery, B.P. 1992, Numerical Recipes in Fortran, Cambridge University Press

Prinn, R.G. \& Fegley, Jr.B. 1989, in The Origin and Evolution of Planetary and Satellite Atmospheres, eds. Atreya, S.K., et al., Univ. of Arizona Press, Tucson, 78

Schleicher, D.G. \& A'Hearn, M.F. 1982, ApJ, 258, 864

Swings, P., Elvey, C.T., \& Babcock, H.W. 1941, ApJ, 94, 320

Tokunaga, A.T., Hanner, M.S., Veeder, G.J., \& A'Hearn, M.F. 1984, AJ, 89, 162

Weaver, H., et al. 1999a, Icarus, in press

Weaver, H., et al. 1999b, Icarus, in press

Weaver, H., et al. 1999c, BAAS, in press

Woodney, L.M., McMullin, J., \& A'Hearn, M.G. 1997, Planetary and Space Sci., 45,717

Wyckoff, S., Fox, R., Wehinger, P., Heyd, R., \& Ferro, A. 1996, BAAS, 28, 1093

\section{Discussion}

E.F. van Dishoeck: Have you or any of your colleagues analyzed the ${ }^{12} \mathrm{C} /{ }^{13} \mathrm{C}$ ratio from the optical data on $\mathrm{C}_{2}$ or $\mathrm{CN}$ ?

S. J. Kim: We have not derived the ${ }^{12} \mathrm{C} /{ }^{13} \mathrm{C}$ ratio in comets. Drs. S. Wyckoff and $\mathrm{M}$. Kleine derived ${ }^{12} \mathrm{C} /{ }^{13} \mathrm{C}$ ratios from high-resolution $\mathrm{CN}$ spectra of comets, and the derived values are close to the terrestrial value.

J. Storey: Do you have spatially-resolved information from long-slit spectroscopy? If so, do you see any evolution of the chemistry as a function of radius from the nucleus?

S. J. Kim: We usually obtain spatially-resolved spectra for various comets using long-slit spectroscopy, and yes, we often see the evolution of the (photo-) chemistry for certain species as a function of cometocentric distance. In particular, a short-lived molecule such as $\mathrm{S}_{2}$ can only be seen very close to the nucleus. Another short-lived molecule, $\mathrm{CH}$, is usually seen up to $10^{5} \mathrm{~km}$ from the nucleus, and this observational fact indicates that $\mathrm{CH}$ should have extended sources and/or long-lived parent molecules. 